\title{
Progress in the improved lattice calculation of direct CP- violation in the Standard Model
}

\author{
Christopher Kelly ${ }^{1}$ \\ ${ }^{1}$ Columbia University, 538 W 120th St., New York NY 10027
}

\begin{abstract}
We discuss the ongoing effort by the RBC \& UKQCD collaborations to improve our lattice calculation of the measure of Standard Model direct CP violation, $\epsilon^{\prime}$, with physical kinematics. We present our progress in decreasing the (dominant) statistical error and discuss other related activities aimed at reducing the systematic errors.
\end{abstract}

\section{Introduction}

The leading explanation for the dominance of matter over antimatter in the Universe, baryogenesis, requires the breaking of the CP-symmetry. While such a breaking occurs in the Standard Model, its size appears insufficient to account for the disparity, suggesting new Beyond the Standard Model sources of CP-violation (CPV) have yet to be discovered.

A particularly attractive avenue for searching for such physics is in the direct CP-violation occuring in the decays of $K_{L}$ into two pions, which is heavily suppressed in the Standard Model. This was discovered during the late 1990s with the following result:

$$
\operatorname{Re}\left(\epsilon^{\prime} / \epsilon\right) \approx \frac{1}{6}\left(1-\left|\frac{\eta_{00}}{\eta_{ \pm}}\right|^{2}\right)=16.6(2.3) \times 10^{-4}
$$

where $\epsilon^{\prime}$ and $\epsilon$ are the measures of direct and indirect CPV, respectively, and $\eta_{i j}=A\left(K_{L} \rightarrow\right.$ $\left.\pi_{i} \pi_{j}\right) / A\left(K_{S} \rightarrow \pi_{i} \pi_{j}\right)$. Unfortunately the use of this ground-breaking discovery in the search for new physics has until recently been hampered by the lack of a corresponding, reliable Standard Model calculation, due to the processes involved receiving large contributions from non-perturbative QCD effects, thus preventing the accurate use of perturbation theory.

In 2015 the RBC \& UKQCD collaborations published [1] the first direct calculation of $\epsilon^{\prime}$ in the Standard Model using lattice QCD via the isospin-definite amplitudes $A_{I}=K \rightarrow(\pi \pi)_{I}$, where $I$ refers to the isospin quantum number of the final $\pi \pi$ state. These amplitudes are computed as

$$
A_{I}=F \frac{G_{F}}{\sqrt{2}} V_{u s}^{*} V_{u d}\left[z_{i}(\mu)+\tau y_{i}(\mu)\right] Z_{i j}(\mu)\left\langle(\pi \pi)_{I}\left|Q_{j}(\mu)\right| K\right\rangle,
$$

where $F$ is the Lellouch-Lüscher factor [2] that represents the finite-volume correction to the decay amplitude, $z$ and $y$ are c-number Wilson coefficients, $\tau=-V_{t s}^{*} V_{t d} / V_{u d} V_{u s}^{*}, V_{i j}$ are CKM matrix elements, and $Q_{i}$ are a set of dimension-six four-quark operators. $Z_{i j}$ is the renormalization matrix 
relating the bare lattice operators to $\overline{\mathrm{MS}}$ operators normalized at the scale $\mu$, thereby matching the scheme used in the calculation of the Wilson coefficients.

We obatained the following result:

$$
\begin{aligned}
\operatorname{Re}\left(\frac{\varepsilon^{\prime}}{\varepsilon}\right) & =\operatorname{Re}\left\{\frac{i \omega e^{i\left(\delta_{2}-\delta_{0}\right)}}{\sqrt{2} \varepsilon}\left[\frac{\operatorname{Im} A_{2}}{\operatorname{Re} A_{2}}-\frac{\operatorname{Im} A_{0}}{\operatorname{Re} A_{0}}\right]\right\} \\
& =1.38(5.15)(4.59) \times 10^{-4}
\end{aligned}
$$

where the errors are statistical and systematic, respectively. Here $\delta_{I}$ are the s-wave $\pi \pi$-scattering phase shifts and $\omega=\operatorname{Re} A_{2} / \operatorname{Re} A_{0}$. This result has roughly three times larger errors than the experimental value, and agrees at the $2.1 \sigma$ level. However the possibility of a tension has generated much excitement within the physics community and has provided strong motivation for improving the calculation.

Since the aforementioned publication we have pursued a programme of substantially improving the errors on our calculation, and in this document we provide an update on our progress.

\section{Brief review of lattice calculation of $A_{0}$}

The errors on our result are dominated by those of $A_{0}$. We begin by briefly reviewing the details of the calculation of this quantity. We performed our measurements on 216 lattice configurations generated with the Möbius domain wall fermion action with $L_{s}=12$ and the Iwasaki+DSDR gauge action at $\beta=2.13$. Our pion masses are physical, measuring $m_{\pi}=143.1(2.0) \mathrm{MeV}$, and the inverse lattice spacing is a somewhat coarse $1.378(7) \mathrm{GeV}$. With this coarse lattice spacing we obtain a large, $(4.6 \mathrm{fm})^{3}$ spatial volume - vital for controlling finite-volume effects - while keeping the number of lattice sites to a computationally tractable number, at the cost of increased discretization errors. Here the Iwasaki+DSDR action heavily suppresses the 'dislocations' (tears) in the gauge field that contribute strongly to the residual chiral symmetry breaking on coarse lattices, allowing us to simulate with a small fifth-dimensional extent.

On the lattice, the ground-state of the $I=0 \pi \pi$ system (after subtracting the vacuum contribution), comprises two pions at rest, and thus has an energy of $\sim 280 \mathrm{MeV}$, much smaller than the kaon mass of $\sim 500 \mathrm{MeV}$. In order to ensure that the $K \rightarrow \pi \pi$ decay is energy conserving we therefore use Gparity boundary conditions (BCs) [3-5] on the quarks in all three spatial directions; these boundary conditions make the pion states antiperiodic in the spatial directions, thereby increasing their groundstate momentum from zero to $\pi / L$, where $L=32$ is the spatial box size. With this choice of volume and boundary condition we obtained a close match between our $\pi \pi$ energy of $E_{\pi \pi}^{\mathrm{I}=0}=498(11) \mathrm{MeV}$ and our kaon mass of $m_{K}=490.6(2.4) \mathrm{MeV}$. Unfortunately the presence of disconnected diagrams requires degeneracy between the valence and sea spectra in order to satisfy unitarity, and as such we must generate new, custom gauge configurations with G-parity BCs.

The measurements were performed using all-to-all (A2A) propagators [6], whereby an approximation to the propagator is generated by combining the low-mode contribution obtained using 900 exact eigenmodes of the Dirac operator (computed using the Lanczos algorithm), and a stochastic approximation to the high-mode contribution. Aside from the ability to maximally sample the source, sink and operator locations, this approach allows us to tune the spatial structure of our sources/sinks to maximize the overlap with the desired state and, in the case of the $\pi \pi$ state, minimize the contribution of the vacuum; in our case we use 1s hydrogen-wavefunction sources with a radius of two lattice sites.

The renormalization matrices are computed without using perturbative QCD at the hadronic scale through the use of an intermediate, non-perturbative 'regularization-invariant momentum scheme' with symmetric kinematics (RI-SMOM) [7, 8], the basis of which comprises the 7 independent dimension-6 four-quark operators $Q_{1}^{\prime}-Q_{7}^{\prime}$ and all relevant dimension-3 and 4 operators. Since our 


\begin{tabular}{lr|lr} 
Description & Error & Description & Error \\
\hline \hline Finite lattice spacing & $12 \%$ & Finite volume & $7 \%$ \\
Wilson coefficients & $12 \%$ & Excited states & $\leq 5 \%$ \\
Parametric errors & $5 \%$ & Operator renormalization & $15 \%$ \\
Unphysical kinematics & $\leq 3 \%$ & Lellouch-Lüscher factor & $11 \%$ \\
\hline \multicolumn{2}{l}{ Total (added in quadrature) } & & $27 \%$ \\
\hline
\end{tabular}

Table 1: Representative, fractional systematic errors for the individual operator contributions to $\operatorname{Re}\left(A_{0}\right)$ and $\operatorname{Im}\left(A_{0}\right)$.

publication we have extended the basis [9] to include the single two-quark dimension-6 operator $G_{1}$ that mixes at $O(\alpha)$. We neglect two other dimension-6 operators that mix at $O\left(\alpha^{2}\right)$, and all dimension5 operators that come with a coefficient of the quark mass as these are expected to be small. We use this scheme to run to a high energy scale at which continuum perturbation theory can be reliably used to match to $\overline{\mathrm{MS}}$. In our case, however, the limit of the lattice cutoff forced us to to use a rather low scale of $1.531 \mathrm{GeV}$ for this matching, resulting in larger perturbative truncation errors.

We obtained the following values for the real and imaginary parts of $A_{0}$ :

$$
\operatorname{Re}\left(A_{0}\right)=4.66(1.00)(1.26) \times 10^{-7} \mathrm{GeV} \quad \text { and } \quad \operatorname{Im}\left(A_{0}\right)=-1.90(1.23)(1.08) \times 10^{-11} \mathrm{GeV},
$$

where the errors are statistical and systematic, respectively; for this calculation these errors are roughly comparable. The breakdown of the systematic errors is reproduced in Table 1. The most significant contributions are due to the perturbative truncation errors associated with the Wilson coefficients and with the RI-SMOM $\rightarrow \overline{\mathrm{MS}}$ matching. The former is large mainly due to the use of perturbation theory in the three-to-four flavor matching, and the latter due to our low renormalization scale. The discretization error is also comparable in size.

In the remainder of this document we describe our ongoing consecutive efforts to reduce both the systematic and statistical errors.

\section{Reduction of statistical errors}

Our goal was to increase the our statistics by a factor of four, to $O(900)$ measurements, thereby reducing the statistical error by $2 \times$, by the end of 2017 . We describe this programme and the improvements in more detail below.

\subsection{Configuration generation}

\subsubsection{Algorithmic improvements}

Simulating with G-parity BCs is computationally expensive due to a necessary factor of two in the cost of applying the explicitly two-flavor Dirac operator, and also the requirement, for a $2+1$ flavor calculation, to take the square-root of the light-quark action $\operatorname{det}\left(D^{\dagger} D\right)$ (and a fourth-root for the strange quarks), which here represents the action of four independent flavors. The use of $D^{\dagger} D$, where $D$ is the Dirac operator, is to ensure the matrix is Hermitian and positive-definite, a requirement of the conjugate gradient (CG) algorithm.

The rooting is performed using the RHMC algorithm [10], for which multi-shift CG is typically used to efficiently evaluate the terms of the rational approximation. This algorithm is more expensive than regular CG due to significant linear algebra overheads and the inability to use mixed-precision restarted methods. The use of RHMC is particularly disadvantageous for the light quarks for which the 


\begin{tabular}{cc|cc}
\hline \hline Source & Determinant computation & Trajectories & Independent configs. \\
\hline Blue Waters & RHMC & $116+69+37+32$ & $24+12+4+3$ \\
KEKSC & RHMC & 441 & 105 \\
BNL & RHMC & 854 & 208 \\
DiRAC & RHMC & 621 & 150 \\
\hline KEKSC & EOFA & $1119+878$ & $274+214$ \\
BNL & EOFA & 987 & 241 \\
Mira & EOFA & 187 & 41 \\
\hline & & & 1276 total
\end{tabular}

Table 2: A summary of our configuration generation, not including the original 216 configurations. In the "trajectories" column, numbers presented in sum indicate the respective sizes of independent streams running on that same machine. Independent configurations are separated by 4 MD time units, and 20 MD time units are discarded at the start of each stream.

large eigenvalue range and the apparent intolerance of the algorithm to finite-precision errors forced us to use a large number of poles with relatively tight stopping conditions. The additional costs also hamper our ability to use the Hasenbusch preconditioning scheme to tune the molecular dynamics, thus requiring smaller MD time steps.

Recently we have been able to bypass the use of RHMC for the light quarks entirely by implementing with high efficiency [11] the "exact one-flavor action" (EOFA) [12], a variant of the domain wall fermion action that represents the determinant of a single quark flavor (two flavors in our case) without the need for rooting. This is achieved by carefully factorizing the domain wall determinant into two pieces that are each Hermitian and positive-definite, allowing them to be inverted directly using regular CG. This technique opened up the ability to further tune the evolution by introducing more Hasenbusch steps, and allowed us to make full use of mixed-precision CG. We could formerly generate one trajectory every 7.7 hours on 512-nodes of BG/Q using the RHMC-based approach; with EOFA and careful tuning we were able to achieve a factor of $4.2 \times$ speed-up, reducing the time to generate a trajectory to just 1.8 hours on the same machine [11].

\subsubsection{Summary of configuration status}

While configuration generation by Markov chain Monte Carlo is necessarily a sequential process, we were able to significantly increase the rate of configuration generation by running multiple independent streams starting from widely separated points of our original, thermalized ensemble. Our previous results suggest an autocorrelation time of $\lesssim 4$ MD time units, therefore for each stream we discard the first 20 trajectories (5 autocorrelation lengths) to ensure independent ensembles.

We performed the bulk of our generation using the BG/Q machines 'KEKSC' at KEK, Japan; 'DiRAC' at Edinburgh, UK; the 'Mira' machine at ALCF; and the BG/Q machines at RBRC/BNL. Here we utilize the CPS framework with the Bagel/BFM library [13] for highly-optimized BG/Q solvers. We also made some use of the Blue Waters (AMD 'Interlagos' processors) using CPS atop the Grid framework [14] for optimal kernel performance.

In Table 2 we give the status of configuration generation at the time of writing. Including the original 216 configurations, the $O(1500)$ resulting configurations represents a factor of seven increase in statistics over the original calculation. This exceeds our original target of 900 configurations by a significant margin. At this point we have halted further configuration generation in favor of performing measurements, which, due to the above improvements are now lagging significantly behind. 


\subsection{Measurements}

Since the time of our original publication we have completely refactored our CPS-based measurement code and have implemented a number of strategies to improve performance on a variety of architectures, with particular emphasis on the BG/Q and Intel "Knight's Landing" (KNL) machines. The measurements comprise three stages: the generation of the eigenvectors using the Lanczos procedure; the $O(1500)$ deflated Dirac operator inversions associated with the (time-spin-color-flavor diluted) high-mode approximation; and finally the A2A contractions themselves.

\subsubsection{Algorithmic improvements}

For the contractions we have implemented a number of improvements including:

- Spatial decomposition of the CPS fermion fields across the SIMD lanes allowing optimal use of QPX intrinsics on BG/Q and AVX512 intrinsics on Intel KNL.

- A hand-coded AVX512 assembly kernel for the most computationally expensive part of the contractions, which achieves over 400 Gflops double-precision performance on KNL. We have also added Grid-based intrinsics kernels for other architectures including BG/Q, resulting in a significant performance gain.

- Optimized distribution of work over nodes and threads for underlying matrix operations and use of highly-tuned MKL, ATLAS BLAS libraries where appropriate.

- An optimized parallel FFT that divides the lattice into one-dimensional strips and spreads this work over nodes and threads to maximize parallelization.

- A flexible structure for adapting to memory constraints, particularly on the BG/Q machines, including a distributed memory model for storing intermediate data with optional temporary disk storage to make use of burst buffers where available.

As a result of these changes we were able to add additional quantities to our measurement programme while keeping the total measurement time the same on 512-nodes of BG/Q. We now measure with a second source smearing - a 2 s hydrogen-wavefunction form with a radius of 2 - and also compute the $\sigma \rightarrow \sigma$ and $\sigma \rightarrow \pi \pi$ matrix elements, where $\sigma$ is a scalar operator with vacuum quantum numbers. As we discuss below, these will provide additional data in the $\pi \pi$ two-point and $K \rightarrow \pi \pi$ three-point calculations as well as a better handle on excited states.

We have also added the option to replace the Lanczos and CG implementations with those in the Grid framework, allowing us to perform highly optimized measurements on other architectures. Within Grid we have implemented several improvements, including a hand-coded intrinsics-based G-parity kernel implementation achieving 330 Gflops single-precision performance on KNL (singlenode), and an implementation of the mixed-precision 'reliable-update' CG approach [15] with halfprecision communications for the majority of the iterations to maximize the use of limited network bandwidth. These improvements have enabled us to efficiently perform a large number of measurements on the Cori I (Intel 'Haswell') and Cori II (KNL) machines at NERSC.

\subsubsection{Status of measurements}

At the time of writing we have performed 774 measurements, not including the original 216; in total roughly $2 / 3$ of the way towards our goal. As a demonstration of our progress, in Figure 1 we show the results of increasing the number of measurements to 841 configurations from the original 216 for $\pi \pi$ effective energy as well as the $Q_{2}$ and $Q_{6}$ matrix elements, which are the leading contributors to the real and imaginary parts of $A_{0}$, respectively. We observe a $2 \times$ reduction in the statistical errors consistent with $\sqrt{N}$-scaling. 

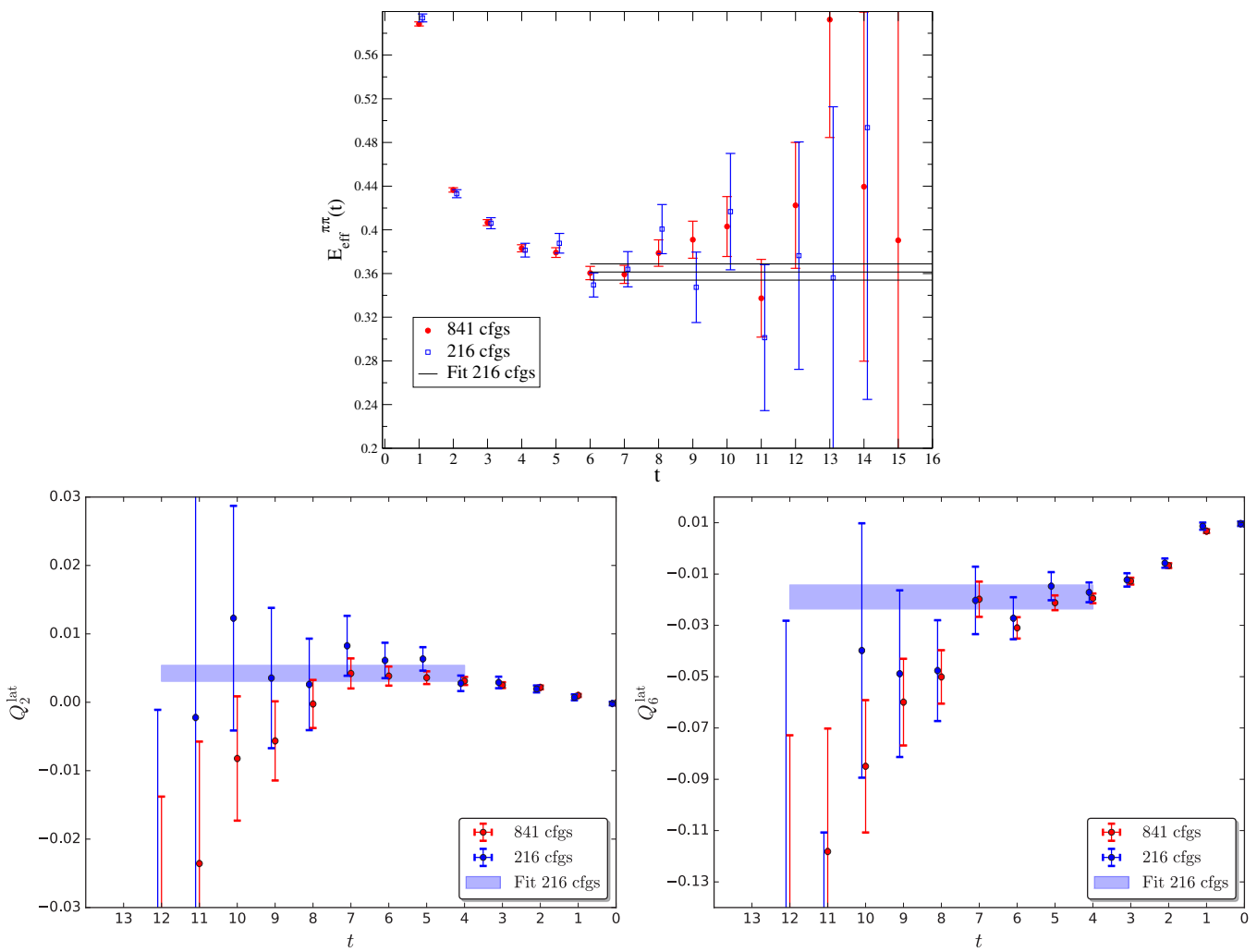

Figure 1: The $\pi \pi$ effective energy (top) and the lattice matrix elements $Q_{2}$ (lower-left) and $Q_{6}$ (lowerright) for 216 configurations and 841 configurations, overlaid by the fit to the former.

\section{Improvements in systematic errors}

\subsubsection{Renormalization and Wilson coefficients}

The $O(15 \%)$ errors on the renormalization arise due to the low, $1.531 \mathrm{GeV}$ scale at which perturbation theory is applied to match between RI-SMOM and $\overline{\mathrm{MS}}$. Since publication we have utilized stepscaling to increase the scale to $2.29 \mathrm{GeV}$, and have also included the two-quark, dimension- 6 operator $G_{1}$ that mixes at $O(\alpha)$, for which the effects are at the percent-scale as expected. These improvements were reported on in Ref. [16], in which we demonstrated a reduction of the renormalization systematic to $8 \%$ (preliminary), along with an improved procedure for determining the error itself.

We do not expect a similar improvement in the error on the Wilson coefficients from increasing the scale because the dominant contribution to the error arises from the use of perturbation theory to cross the charm threshold at $1.29 \mathrm{GeV}$. In order to address this issue without performing a full four-flavor calculation of the matrix elements, it is necessary to non-perturbatively match across this threshold, which requires the computation of non-perturbative renormalization factors at scales much lower than the charm scale. As RI-SMOM is a gauge-fixed scheme there is an enlarged set of gauge-noninvariant operators that mix at higher orders of $\alpha$, and as such these schemes may become unreliable at such low scales. To avoid this issue we are investigating the use of the gauge-invariant position space renormalization scheme, and results thus far seem promising. 


\subsubsection{Finite lattice-spacing errors}

We estimate that our coarse, $1.38 \mathrm{GeV}$ lattice spacing gives rise to $O(12 \%)$ discretization effects, which will dominate the error of our forthcoming calculation. Ideally we would address this by repeating the calculation at a finer lattice spacing, but unfortunately the computational cost of such a venture makes it unfeasible with the present generation of computing hardware.

An alternative is to consider a coarser lattice spacing: For other purposes we have generated a (non-G-parity) $24^{3} \times 64 \times 24$ Möbius DWF ensemble with the Iwasaki+DSDR gauge action at $\beta=1.633$ - corresponding to $a^{-1} \approx 1.0 \mathrm{GeV}$ - and a physical pion mass. This lattice, unintentionally, has an almost identical physical spatial volume to our $32^{3} \times 64$ G-parity ensemble, making it an ideal candidate for a repeat calculation. While this lattice is very coarse compared to a typical lattice calculation, it appears that the Iwasaki+DSDR gauge action has remarkably small discretization errors, at the percent-scale on this $1 \mathrm{GeV}$ ensemble for a variety of quantities [17]. We must of course generate new configurations with G-parity BCs, but the existence of the non-G-parity lattice enables us to easily compute the RI-SMOM renormalization matrices and the lattice spacing without the complications associated with the G-parity BCs (a situation we also took advantage of in our previous calculation).

While further study of the discretization effects on other, more complex quantities such as $B_{K}$ and $A_{2}$ are underway, we have thermalized an ensemble with G-parity BCs in anticipation of proceeding with this calculation.

\subsubsection{Resolving the " $\pi \pi$ puzzle"}

Our previous result for the $I=0 \pi \pi$ scattering phase shift, computed as part of the $A_{0}$ calculation, is $\delta_{0}=23.8(4.9)(1.2)^{\circ}$ [1], where the errors are statistical and systematic, respectively. This value is somewhat lower than the value of $38.3(1.3)^{\circ}[18,19]$ obtained by combining experimental data with the Roy equations. In order to match this phase shift, our $\pi \pi$ energy would need to be $\sim 470 \mathrm{MeV}$ as opposed to the 498(11) MeV measured value.

To address this issue, along with the increased statistics we now measure pion operators with a 2s hydrogen-wavefunction smearing and also compute the $\sigma \rightarrow \sigma$ and $\sigma \rightarrow \pi \pi$ two-point functions. In the continuum the $\sigma$ is a broad resonance of the $I=0 \pi \pi$ system, but on the lattice it is simply a state that mixes with the $\pi \pi$; the eigenstate of the finite volume QCD Hamiltonian that we refer to as the " $\pi \pi$ " is therefore more precisely a linear superposition of these two states. While the finitevolume dynamics of this state in the scattering and decays are completely captured by the Lüscher and Lellouch-Lüscher formalisms, respectively, studying correlation functions involving this operator provides more handles on the $\pi \pi$ system with differing excited-state contributions, and therefore offers not just an improvement in statistics on the ground-state energy but also a greater ability to study the spectrum of nearby states, particularly if coupled with the variational method [20]. This will help us to resolve whether our appparently too-large $\pi \pi$ energy is due to contamination from a nearby excited state (although such a state would be incompatible with phenomenology).

\subsubsection{Other related, future projects}

We have also initiated a number of related long-term projects. One such is the forthcoming calculation of the $I=0 \pi \pi$ energy using traditional periodic boundary conditions and modern, multi-operator techniques to extract the excited states. We are also examining the possibility of computing $K \rightarrow$ $\pi \pi$ including isospin-breaking and electromagnetic effects [21], and studying the non-perturbative computation of the Wilson coefficients [22], both of which were reported on at this conference. 


\section{Conclusions}

In these proceedings we have detailed our progress in the improved calculation of $A_{0}$, and therefore $\epsilon^{\prime}$, on the lattice. We report significant progress towards a seven-fold increase in statistics over our initial calculation, with the majority of the work having been completed at the time of writing.

We also discussed a number of programmes to improve the systematic errors, including the addition of new $\pi \pi$ operators to better resolve the ground-state energy; a factor of $2 \times$ reduction in the renormalization systematic as a result of step-scaling to a higher energy scale; and the inclusion of the $G_{1}$ operator in the renormalization. In addition we outlined our plan for a calculation of $A_{0}$ with a second, coarser lattice spacing in order to better constrain the discretization errors. Finally we mentioned several longer-term projects such as computing the $3 \rightarrow 4$ flavor matching non-perturbatively using position-space NPR; studying the effects of electromagnetism and isospin breaking; and a calculation of the $\pi \pi$ energy using periodic boundary conditions and multi-operator methods.

It is our intention to publish new results for $A_{0}$ and $\epsilon^{\prime}$ in early 2018.

\section{References}

[1] Z. Bai et al. (RBC, UKQCD), Phys. Rev. Lett. 115, 212001 (2015), 1505.07863

[2] L. Lellouch, M. Luscher, Commun. Math. Phys. 219, 31 (2001), hep-lat/0003023

[3] U.J. Wiese, Nucl. Phys. B375, 45 (1992)

[4] C.h. Kim, N.H. Christ, Nucl. Phys. Proc. Suppl. 119, 365 (2003), [,365(2002)], hep-lat/0210003

[5] C. Kelly (RBC, UKQCD), PoS LATTICE2012, 130 (2012)

[6] J. Foley, K. Jimmy Juge, A. O'Cais, M. Peardon, S.M. Ryan, J.I. Skullerud, Comput. Phys. Commun. 172, 145 (2005), hep-lat/0505023

[7] G. Martinelli, C. Pittori, C.T. Sachrajda, M. Testa, A. Vladikas, Nucl. Phys. B445, 81 (1995), hep-lat $/ 9411010$

[8] C. Sturm, Y. Aoki, N.H. Christ, T. Izubuchi, C.T.C. Sachrajda, A. Soni, Phys. Rev. D80, 014501 (2009), 0901.2599

[9] G. McGlynn (2016), 1605.08807

[10] M.A. Clark, A.D. Kennedy, Nucl. Phys. Proc. Suppl. 129, 850 (2004), [,850(2003)], hep-lat/0309084

[11] C. Jung, C. Kelly, R.D. Mawhinney, D.J. Murphy (2017), 1706.05843

[12] Y.C. Chen, T.W. Chiu (TWQCD), Phys. Lett. B738, 55 (2014), 1403.1683

[13] P.A. Boyle, Comput. Phys. Commun. 180, 2739 (2009)

[14] P.A. Boyle, G. Cossu, A. Yamaguchi, A. Portelli, PoS LATTICE2015, 023 (2016)

[15] G.L.G. Sleijpen, H.A. van der Vorst, Computing 56, 141 (1996)

[16] C. Kelly, PoS LATTICE2016, 308 (2016)

[17] R.D. Mawhinney, EPJ Web Conf. LATTICE2017 (2017)

[18] G. Colangelo, J. Gasser, H. Leutwyler, Nucl. Phys. B603, 125 (2001), hep-ph/0103088

[19] G. Colangelo, personal communication

[20] M. Luscher, U. Wolff, Nucl. Phys. B339, 222 (1990)

[21] N.H. Christ, EPJ Web Conf. LATTICE2017 (2017)

[22] M. Bruno, EPJ Web Conf. LATTICE2017 (2017) 